\title{
Strategic disclosure of random variables.
}

Citation for published version (APA):

Flesch, J., \& Perea ý Monsuwé, A. (2009). Strategic disclosure of random variables. Kwantitatieve Economie. METEOR Research Memorandum No. 023 https://doi.org/10.26481/umamet.2009023

Document status and date:

Published: 01/01/2009

DOI:

10.26481/umamet.2009023

Document Version:

Publisher's PDF, also known as Version of record

\section{Please check the document version of this publication:}

- A submitted manuscript is the version of the article upon submission and before peer-review. There can be important differences between the submitted version and the official published version of record.

People interested in the research are advised to contact the author for the final version of the publication, or visit the DOI to the publisher's website.

- The final author version and the galley proof are versions of the publication after peer review.

- The final published version features the final layout of the paper including the volume, issue and page numbers.

Link to publication

\footnotetext{
General rights rights.

- You may freely distribute the URL identifying the publication in the public portal. please follow below link for the End User Agreement:

www.umlib.nl/taverne-license

Take down policy

If you believe that this document breaches copyright please contact us at:

repository@maastrichtuniversity.nl

providing details and we will investigate your claim.
}

Copyright and moral rights for the publications made accessible in the public portal are retained by the authors and/or other copyright owners and it is a condition of accessing publications that users recognise and abide by the legal requirements associated with these

- Users may download and print one copy of any publication from the public portal for the purpose of private study or research.

- You may not further distribute the material or use it for any profit-making activity or commercial gain

If the publication is distributed under the terms of Article $25 \mathrm{fa}$ of the Dutch Copyright Act, indicated by the "Taverne" license above, 


\section{Maastricht University}

János Flesch, Andrés Perea

Strategic Disclosure of Random Variables

$\mathrm{RM} / 09 / 023$

\section{METEOR}

Faculty of Economics and Business Administration Maastricht Research School of Economics

of Technology and Organization

P.O. Box 616

NL - 6200 MD Maastricht

The Netherlands 


\title{
Strategic Disclosure of Random Variables*
}

\author{
János Flesch and Andrés Perea ${ }^{\dagger}$
}

June 2, 2009

\begin{abstract}
We consider a game $G_{n}$ played by two players. There are $n$ independent random variables $Z_{1}, \ldots, Z_{n}$, each of which is uniformly distributed on $[0,1]$. Both players know $n$, the independence and the distribution of these random variables, but only player 1 knows the vector of realizations $\mathbf{z}:=\left(z_{1}, \ldots, z_{n}\right)$ of them. Player 1 begins by choosing an order $z_{k_{1}}, \ldots, z_{k_{n}}$ of the realizations. Player 2, who does not know the realizations, faces a stopping problem. At period 1 , player 2 learns $z_{k_{1}}$. If player 2 accepts, then player 1 pays $z_{k_{1}}$ euros to player 2 and play ends. Otherwise, if player 2 rejects, play continues similarly at period 2 with player 1 offering $z_{k_{2}}$ euros to player 2. Play continues until player 2 accepts an offer. If player 2 has rejected $n-1$ times, player 2 has to accept the last offer at period $n$. This model extends Moser's (1956) problem, which assumes a non-strategic player 1.

We examine different types of strategies for the players and determine their guaranteelevels. Although we do not find the exact value $v_{n}$ of the game $G_{n}$ in general, we provide an interval $I_{n}=\left[a_{n}, b_{n}\right]$ containing $v_{n}$ such that the length of $I_{n}$ is at most 0.07 and converges to 0 as $n$ tends to infinity. We also point out strategies, with a relatively simple structure, which guarantee that player 1 has to pay at most $b_{n}$ and player 2 receives at least $a_{n}$. In addition, we completely solve the special case $G_{2}$ where there are only two random variables. We mention a number of intriguing open questions and conjectures, which may initiate further research on this subject.
\end{abstract}

Key words: Secretary problem, Moser's problem, incomplete information, lack of information on one side, optimal strategies.

\section{Introduction}

For many years, scientists from different disciplines have explored the well-known "secretary problem". This is a stopping problem in which $n$ secretaries are invited, in a random order, for

\footnotetext{
${ }^{*}$ We thank Péter Csóka and Jérôme Renault for discussions on this subject. We are also grateful to Wolfram Research, Inc., for program package Mathematica, which assisted us with some numerical approximations.

${ }^{\dagger}$ Address of both authors: Department of Quantitative Economics, Maastricht University, P.O. Box 616, 6200 MD Maastricht, The Netherlands. Email: J.Flesch@maastrichtuniversity.nl and A.Perea@maastrichtuniversity.nl.
} 
an interview to fill a secretarial position. The employer knows the number of secretaries, and is aware that the order is random. After every interview, the employer can rank the secretaries interviewed so far from best to worst without ties, and must decide whether or not to hire the last candidate. His task is to find a stopping rule that maximizes the probability of hiring the best secretary. The optimal stopping rule has the following form: Reject the first $r_{n}$ secretaries, and then hire the first secretary who is better than all the preceding ones. If no such secretary arrives after round $r_{n}$, then the best candidate was among the first $r_{n}$ secretaries, and it therefore does not make a difference whether or not to hire the last secretary. For large $n$, the optimal choice of $r_{n}$ is approximately $n / e$, and the probability of hiring the best secretary is approximately $1 / e$. For a historical overview of this classical secretary problem the reader is referred to Ferguson (1989).

The secretary problem has been extended in a number of important directions. In particular, versions have been studied in which the payoff depends on the rank of the selected candidate, even if he or she is not the best. This seems more realistic than the classical scenario, as hiring the second best candidate is obviously better than hiring the third best. We can further extend this situation by assuming that every secretary has a cardinal value distributed according to some probability measure, but where the payoff solely depends on the rank of the selected candidate (see Gnedin and Krengel (1995) and the references therein, and Bearden (2006)). In this case, however, it is perhaps more natural to assume that the payoff is exactly equal to the cardinal value of the selected candidate, instead of its relative rank. For instance, if there are two secretaries with neigbouring ranks, then selecting the best amongst these two is less relevant if their values are close, and more relevant if the difference in values is high. This is exactly the model as studied by Moser (1956), who assumes that the values are independently and uniformly distributed on $[0,1]$. In fact, Moser's model is a variant of a problem considered by Arthur Cayley in the nineteenth century. (See Ferguson (1989) for a description of Cayley's problem.)

In the present paper we take Moser's model, but assume in addition that there is an adversary who knows the values of the secretaries, and chooses the order of the secretaries strategically. The employer, on the other hand, does not know these values, but only knows the number of secretaries, and the distribution of their respective values. We thus obtain a zero-sum game with incomplete information on the employer's side. We are not the first to take a game theoretic approach to the problem ${ }^{1}$. See, for instance, Gilbert and Mosteller (1966), Gnedin and Krengel (1995), and de Carvalho, Chaves, de Abreu Silva (2008).

The adversary's main problem is how to optimally exploit his private information. This is a difficult problem since the adversary, by using his private information, would make choices that would reveal part of his private information to the employer. In our analysis, however, we mainly focus on the employer, in line with the literature on the secretary problem. In particular, we

\footnotetext{
${ }^{1}$ Most of the models are interested in the relative rank of the chosen secretary, and not in the cardinal value as we are. There is, however, a relationship between the two approaches. Bruss and Ferguson (1993) show, namely, that there is a strong correlation between the cardinal values and their associated ranks. See also Bruss (2005).
} 
will be interested in his optimal strategies, that is, strategies for which the worst-case expected payoff is as high as possible.

For the case of two secretaries, we show that the employer's unique ${ }^{2}$ optimal strategy is to hire the first secretary precisely when her value is at least 0.5 . This strategy guarantees an expected payoff of $7 / 12$ to the employer. An optimal strategy for the adversary is to first send the secretary whose value is closer to 0.5 .

If there are more than two secretaries, we are not able to find exact optimal strategies for the employer. However, we provide strategies with a simple structure that approach the value within a distance of at most 0.07 . The class of strategies we focus on are threshold strategies, and they work as follows: For every period $k$ choose a threshold $a_{k}$, which may depend on the values of the rejected secretaries, and hire the current secretary precisely when her value is at least $a_{k}$. Such a threshold strategy is called stationary if $a_{k}$ is constant throughout the game, except for the last period where the employer must accept the last candidate. The strategy is a Markov threshold strategy if $a_{k}$ depends on the period $k$, but not on the values of the rejected secretaries.

We show that the best stationary threshold strategy is to choose the threshold equal to $(1 / n)^{1 /(n-1)}$, where $n$ is the number of secretaries. This threshold converges (slowly) to 1 if $n$ tends to infinity. Interestingly, this is also the best stationary threshold strategy in Moser's model, where the order of the secretaries is not chosen strategically. We show that this stationary threshold strategy performs relatively well in general, as it approximates the value by at most 0.08 .

We then turn to Markov threshold strategies. We show that the best amongst these involves thresholds that are non-increasing over time. For the case of two and three secretaries, this strategy is in fact the best stationary threshold strategy discussed above. So, for these cases choosing different thresholds over time does not yield higher payoffs. We conjecture, supported by numerical simulations, that this remains to be true for more than three secretaries as well.

However, for at least three secretaries, we show that the best threshold strategy must base its thresholds not only on the period, but also on the values of the rejected secretaries. Nevertheless, it remains true that the thresholds should be non-increasing over time. We prove that the employer, by using such general threshold strategies, can approach the value by at most 0.07 .

It turns out to be very difficult to provide effective strategies for the adversary. We do, however, provide some suggestions at the end of the paper.

The outline of the paper is as follows: In Section 2 we introduce the model. In Section 3 we describe the optimal strategy for the employer if the order of secretaries is not chosen strategically. After this section we will explore the situation where the adversary is strategic, that is, chooses the order of secretaries to his own advantage. Section 4 covers the case of two secretaries. In Section 5 we turn to the case of more than two secretaries, and examine stationary threshold strategies for the employer. General threshold strategies are explored in Section 6 .

\footnotetext{
${ }^{2}$ To be precise, unique up to behavior on a set of measure zero.
} 
Section 7 contains some concluding remarks, also on effective strategies for the adversary. Some technical proofs have been moved to the appendix.

\section{The Model}

The game. Consider the following game $G_{n}$, where $n \in \mathbb{N}$, played by two players. There are $n$ independent random variables $Z_{1}, \ldots, Z_{n}$, each of which is uniformly distributed on $[0,1]$. We assume that both players know $n$, the independence and the distribution of these random variables, but only player 1 knows the vector of realizations $\mathbf{z}:=\left(z_{1}, \ldots, z_{n}\right)$ of them. The game is played as follows. Let $N=\{1, \ldots, n\}$. At period 1 , player 1 chooses one of $\left\{z_{i}\right\}_{i \in N}$, say $z_{k_{1}}$, and offers $z_{k_{1}}$ euros to player 2 . If player 2 accepts, then player 1 pays $z_{k_{1}}$ euros to player 2 and play ends. Otherwise, if player 2 rejects, play continues at period 2 , where player 1 chooses one of the remaining amounts $\left\{z_{i}\right\}_{i \in N-\left\{k_{1}\right\}}$, say $z_{k_{2}}$. Player 1 subsequently offers $z_{k_{2}}$ euros to player 2 , who either accepts or rejects. If player 2 accepts, then player 1 pays $z_{k_{2}}$ euros to player 2 and play ends, whereas if player 2 rejects, then player 1 has to offer one of the remaining amounts $\left\{z_{i}\right\}_{i \in N-\left\{k_{1}, k_{2}\right\}}$. This continues until player 2 accepts an offer. If player 2 has rejected $n-1$ times, player 2 has to accept the last offer at period $n$.

In terms of the secretary problem as described in the introduction, player 1 corresponds to the adversary whereas player 2 plays the role of employer. The realizations of the random variables $Z_{1}, \ldots, Z_{n}$ are the values of the $n$ secretaries.

Strategies. Let $\phi(N)$ denote the set of all permutations of $N$. Note that player 1's pure strategy is essentially the same as just chosing one permutation in $\phi(N)$ in advance, instead of choosing period by period, and to offer $z_{\phi(k)}$ at period $k$. A (mixed) strategy $\sigma^{1}$ for player 1 is a decision rule which specifies a probability distribution on $\phi(N)$ for each possible vector of realizations $\mathbf{z} .^{3}$ A strategy $\sigma^{1}$ is called pure if, for any $\mathbf{z}$, it prescribes one specific permutation with probability 1.

At any decision point for player 2, the history observed by player 2 is the sequence consisting of all amounts that player 1 has offered before the current period. A (mixed) strategy $\sigma^{2}$ for player 2 is a decision rule which assignes a probability distribution on $\{$ Accept, Reject $\}$ to any offer at the current period and to any history of player $2{ }^{4}$ A strategy $\sigma^{2}$ is called pure if, for any current offer and any history of player 2, strategy $\sigma^{2}$ prescribes either Accept with probability 1 or Reject with probability 1 . Moreover, a pure strategy $\sigma^{2}$ for player 2 is called a threshold strategy if, after any history $h$ of player 2 , there exists a threshold $a(h) \in[0,1]$ such that $\sigma^{2}$ prescribes to accept the current offer $y$ when $y \geq a(h)$ and prescribes to reject it when $y<a(h)$. If these thresholds only depend on the period, then $\sigma^{2}$ is called a Markov threshold

\footnotetext{
${ }^{3}$ We assume throughout that $\sigma^{1}$ satisfies a standard measurability requirement with respect to the Lebesgue $\sigma$-algebra on $[0,1]^{n}$.

${ }^{4}$ Again, we assume that $\sigma^{2}$ is measurable with respect to the Lebesgue $\sigma$-algebra.
} 
strategy, whereas if there is just one threshold then $\sigma^{2}$ is called a stationary threshold strategy. ${ }^{5}$ Markov threshold strategies for player 2 are given and denoted by the sequence of thresholds $\mathbf{a}:=\left(a_{1}, \ldots, a_{n-1}\right)$ for the first $n-1$ periods, whereas a stationary threshold strategy of player 2 is simply a threshold $a \in[0,1]$.

Utility. With respect to a pair of strategies $\left(\sigma^{1}, \sigma^{2}\right)$, let $U\left(\sigma^{1}, \sigma^{2}\right)$ denote the expected amount that player 1 has to pay to player 2 . We also refer to $U\left(\sigma^{1}, \sigma^{2}\right)$ as the expected utility. We evaluate every strategy $\sigma^{1}$ of player 1 by

$$
\psi^{1}\left(\sigma^{1}\right)=\sup _{\sigma^{2}} U\left(\sigma^{1}, \sigma^{2}\right),
$$

which is the worst-case scenario for what player 1 has to pay in expectation. Similarly, for every strategy $\sigma^{2}$ of player 2 , let

$$
\psi^{2}\left(\sigma^{2}\right)=\inf _{\sigma^{1}} U\left(\sigma^{1}, \sigma^{2}\right) .
$$

A strategy $\sigma^{1}$ for player 1 is called a best reply to a strategy $\sigma^{2}$ of player 2 , if $U\left(\sigma^{1}, \sigma^{2}\right)=$ $\psi^{2}\left(\sigma^{2}\right)$. Similarly, a strategy $\sigma^{2}$ for player 2 is called a best reply to a strategy $\sigma^{1}$ of player 1 , if $U\left(\sigma^{1}, \sigma^{2}\right)=\psi^{1}\left(\sigma^{1}\right)$. Best replies always exist in pure strategies.

The value. We always have

$$
\inf _{\sigma^{1}} \psi^{1}\left(\sigma^{1}\right) \geq \sup _{\sigma^{2}} \psi^{2}\left(\sigma^{2}\right) .
$$

If they are equal, then this amount is called the value of the game, and is denoted by $v_{n}$. If $v_{n}$ exists, then a strategy $\sigma^{1}$ for player 1 is called optimal if $\psi^{1}\left(\sigma^{1}\right)=v_{n}$, whereas a strategy $\sigma^{2}$ for player 2 is called optimal if $\psi^{2}\left(\sigma^{2}\right)=v_{n}$. Note that $\sigma^{1}$ and $\sigma^{2}$ are optimal if and only if they are best replies to each other.

One can show the following theorem based on approximating the original game by a sequence of finite discretizations.

Theorem 2.1. (Existence of value and optimal strategies)

The value $v_{n}$ of the game $G_{n}$ exists. Moreover, both players have an optimal strategy.

\section{Playing Against a Non-Strategic Player 1}

In this section, we examine the situation in which player 1 does not manipulate the order of the realizations $z_{1}, \ldots, z_{n}$, and simply chooses $z_{k}$ for period $k$. For every $n$, let $\tau_{n}^{1}$ denote this strategy for player 1 , and let $\widetilde{v}_{n}$ denote the best utility player 2 can achieve against $\tau_{n}^{1}$, i.e. $\widetilde{v}_{n}=\psi^{1}\left(\tau_{n}^{1}\right)$.

\footnotetext{
${ }^{5}$ In accordance with the literature on dynamic games, we use the term Markov to emphasize that the thresholds only depend on the current period but not on the specific history. Similarly, we use stationary to emphasize the time and history independence of the thresholds.
} 
The most important properties of this situation are summarized below. Most of these were already proven by Moser (1956).

Theorem 3.1. (Non-strategic player 1)

(1) Player 2's best reply to $\tau_{n}^{1}$, unique up to a set of measure zero, is the Markov threshold strategy which, for period $k \in\{1, \ldots, n\}$, prescribes threshold $b_{k}:=\widetilde{v}_{n-k}$. (Recall that $\widetilde{v}_{n-k}$ is player 2's best utility against $\left.\tau_{n-k}^{1}\right)$.

(2) Player 2's best utility $\widetilde{v}_{n}$ satisfies the recursion $\widetilde{v}_{1}=\frac{1}{2}$, and $\widetilde{v}_{n}=\frac{1}{2}+\frac{1}{2}\left(\widetilde{v}_{n-1}\right)^{2}$ for all $n \geq 2$.

(3) The sequence $\widetilde{v}_{n}$ is strictly increasing and $\lim _{n \rightarrow \infty} \widetilde{v}_{n}=1$.

(4) Player 2's best amongst the stationary threshold replies to $\tau_{n}^{1}$ is $a_{n}^{*}=\left(\frac{1}{n}\right)^{\frac{1}{n-1}}$, i.e. for any stationary threshold strategy a for player 2 we have $U\left(\tau_{n}^{1}, a_{n}^{*}\right) \geq U\left(\tau_{n}^{1}, a\right)$. The strategy $a_{n}^{*}$, while not being a best reply to $\tau_{n}^{1}$ for any $n \geq 3$, is asymptotically a best reply, i.e. $\lim _{n \rightarrow \infty} U\left(\tau_{n}^{1}, a_{n}^{*}\right)=\lim _{n \rightarrow \infty} \widetilde{v}_{n}(=1)$.

Proof. First, we show part 1. Consider period 1. If player 2 decides to reject, then $n-1$ amounts will remain, yielding $\widetilde{v}_{n-1}$ in expectation at a best continuation. Hence, if player 2 is offered at least $\widetilde{v}_{n-1}$ at period 1 , then he accepts, otherwise he rejects. This argument holds for any later period, which proves part 1.

Next, we prove part 2. At period 1, with regard to the strategy prescribed in part 1, player 2 accepts with probability $1-\widetilde{v}_{n-1}$, with conditional expected amount $\frac{1}{2}\left(\widetilde{v}_{n-1}+1\right)$, and rejects with probability $\widetilde{v}_{n-1}$. Therefore,

$$
\widetilde{v}_{n}=\left(1-\widetilde{v}_{n-1}\right) \cdot \frac{1}{2}\left(\widetilde{v}_{n-1}+1\right)+\widetilde{v}_{n-1} \cdot \widetilde{v}_{n-1}=\frac{1}{2}+\frac{1}{2}\left(\widetilde{v}_{n-1}\right)^{2} .
$$

It is obvious that $\widetilde{v}_{1}=\frac{1}{2}$, so part 2 has been verified.

Part 3 is simple and intuitive. Take some $n \geq 2$. Then, $\widetilde{v}_{n}>\widetilde{v}_{n-1}$ because by (3.1)

$$
\widetilde{v}_{n}=\left(1-\widetilde{v}_{n-1}\right) \cdot \frac{1}{2}\left(1+\widetilde{v}_{n-1}\right)+\widetilde{v}_{n-1} \cdot \widetilde{v}_{n-1}>\left(1-\widetilde{v}_{n-1}\right) \cdot \widetilde{v}_{n-1}+\widetilde{v}_{n-1} \cdot \widetilde{v}_{n-1}=\widetilde{v}_{n-1} .
$$

Here we used that $\widetilde{v}_{n-1} \in(0,1)$. Since the sequence $\widetilde{v}_{n}$ is strictly increasing and $\widetilde{v}_{n} \leq 1$ for all $n$, we may conclude that $\lim _{n \rightarrow \infty} \widetilde{v}_{n}$ exists. By part 2 ,

$$
\lim _{n \rightarrow \infty} \widetilde{v}_{n}=\frac{1}{2}+\frac{1}{2} \lim _{n \rightarrow \infty}\left(\widetilde{v}_{n}\right)^{2},
$$

yielding $\lim _{n \rightarrow \infty} \widetilde{v}_{n}=1$.

Finally, we show part 4 . Take a stationary threshold strategy $a$ for player 2 . With probability $a^{n-1}$, we have $z_{i}<a$ for all $i \in\{1, \ldots, n-1\}$, in which case player 2 rejects all $z_{1}, \ldots, z_{n-1}$ and must accept $z_{n}$, yielding a conditional expectation of $\frac{1}{2}$. On the other hand, with probability 
$1-a^{n-1}$, we have $z_{i} \geq a$ for at least one $i \in\{1, \ldots, n-1\}$, hence player 2 will accept the first amount above $a$, yielding a conditional expectation of $\frac{1}{2}(a+1)$. Thus, strategy $a$ gives

$$
U\left(\tau_{n}^{1}, a\right)=a^{n-1} \cdot \frac{1}{2}+\left(1-a^{n-1}\right) \cdot \frac{1}{2}(a+1)=\frac{1}{2}\left(1+a-a^{n}\right) .
$$

By taking derivatives, it easily follows that $U\left(\tau_{n}^{1}, a\right)$ has a unique maximum at $a_{n}^{*}=\left(\frac{1}{n}\right)^{\frac{1}{n-1}}$, which is in $[0,1]$.

Note that

$$
U\left(\tau_{n}^{1}, a_{n}^{*}\right)=\frac{1}{2}\left(1+a_{n}^{*}-\frac{1}{n} a_{n}^{*}\right),
$$

which, in view of lemma 8.2 , implies

$$
\lim _{n \rightarrow \infty} U\left(\tau_{n}^{1}, a_{n}^{*}\right)=\frac{1}{2}\left(1+\lim _{n \rightarrow \infty} a_{n}^{*}\right)=1 .
$$

Hence, $a_{n}^{*}$ is an asymptotically best reply to $\tau_{n}^{1}$.

Finally, it is clear in view of parts 1 and 3 that $a_{n}^{*}$ is not optimal when $n \geq 3$, since in this case different thresholds must be used at periods 1 and 2 .

Remark: The following table shows an approximation of $\widetilde{v}_{n}$ for some values of $n$ :

\begin{tabular}{|c|c|c|c|c|c|c|c|c|}
\hline$n$ & 2 & 3 & 4 & 5 & 10 & 20 & 50 & 100 \\
\hline$\widetilde{v}_{n}$ & $\approx 0.63$ & $\approx 0.70$ & $\approx 0.74$ & $\approx 0.78$ & $\approx 0.86$ & $\approx 0.92$ & $\approx 0.96$ & $\approx 0.98$ \\
\hline
\end{tabular}

In fact, Moser (1956) and Gilbert and Mosteller (1966) showed that

$$
\widetilde{v}_{n} \approx 1-\frac{2}{n+\ln (n)+b}
$$

if $n$ is large. Here, $b$ is a constant approximately equal to 1.7680 .

\section{The Special Case of Two Random Variables (The Game $G_{2}$ )}

From now on, we will focus on the situation in which there is a strategic adversary. In this section, we examine in detail the case when we have two random variables, that is, $n=2$. So, player 1 must choose an order for the realizations $z_{1}$ and $z_{2}$. In this game both players only have to make a choice at period 1 . Therefore, whenever we speak about a player's choice we always mean his choice at period 1 . We show the following results.

Theorem 4.1. (Properties of the game $G_{2}$ )

(1) An optimal strategy for player 1 is to choose (in period 1) the amount closer to $\frac{1}{2}$, i.e. to choose $z_{1}$ if $\left|z_{1}-\frac{1}{2}\right| \leq\left|z_{2}-\frac{1}{2}\right|$, and to choose $z_{2}$ otherwise.

(2) Player 2's optimal strategy, unique up to a set of measure zero, is the stationary threshold strategy $a=\frac{1}{2}$.

(3) The value is $v_{2}=\frac{7}{12}$. 
Proof. Let $\sigma^{1}$ and $a$ denote the strategies described in part 1 and part 2 , respectively. It is sufficient to show that (1) $\sigma^{1}$ is a best reply to $a,(2) a$ is a best reply to $\sigma^{1}$, unique up to a set of measure zero, and (3) the induced expected utility is $U\left(\sigma^{1}, a\right)=\frac{7}{12}$. In the following, let $x_{1}:=\min \left\{z_{1}, z_{2}\right\}$ and $x_{2}:=\max \left\{z_{1}, z_{2}\right\}$.

Step 1: We show that $\sigma^{1}$ is a best reply to $a$. We distinguish the following cases (we assume that $x_{1} \neq x_{2}$, otherwise player 1's strategy is surely a best reply):

Case 1: $x_{1}<x_{2}<\frac{1}{2}$. In this case, $\sigma^{1}$ offers $x_{2}$, which $a$ rejects, yielding $x_{1}$ as the outcome, which is the best possible amount for player 1 .

Case 2 : $\frac{1}{2} \leq x_{1}<x_{2}$. In this case, $\sigma^{1}$ offers $x_{1}$, which $a$ accepts, yielding $x_{1}$ as the outcome, which is the best possible amount for player 1 .

Case 3: $x_{1}<\frac{1}{2} \leq x_{2}$. In this case, $\sigma^{1}$ offers either $x_{1}$ or $x_{2}$, depending on the exact values of $x_{1}$ and $x_{2}$, but $a$ is going to reject $x_{1}$ and accept $x_{2}$. Thus, the outcome is $x_{2}$. Observe that player 1 cannot achieve $x_{1}$ given player 2's threshold strategy $a=\frac{1}{2}$.

In conclusion, $\sigma^{1}$ is a best reply to $a$ in all cases.

Step 2: We show that $a$ is a best reply to $\sigma^{1}$. It will be clear from the proof that the best reply is unique up to a set of measure zero. Suppose player 1 offers some amount $x$ (which is either $x_{1}$ or $x_{2}$ ). Let $y$ denote the other amount.

Assume first that $x \geq \frac{1}{2}$. Given player 1's strategy, either $y \leq 1-x$ or $y \geq x$, otherwise player 1 would offer $y$ instead of $x$. Thus, given $x$ is offered, $y$ is uniformly distributed over $[0,1-x] \cup[x, 1]$, and therefore has conditional expectation $\frac{1}{2}$.

Assume now that $x<\frac{1}{2}$. Given $x$ is offered, we obtain similarly that $y$ is uniformly distributed over $[0, x] \cup[1-x, 1]$, and therefore has conditional expectation $\frac{1}{2}$ again.

Hence, we see that the conditional expectation of $y$ is always $\frac{1}{2}$, and therefore a best reply for player 2 is to accept $x$ if and only if $x \geq \frac{1}{2}$, in accordance with $a$.

Step 3: We prove that $U\left(\sigma^{1}, a\right)=\frac{7}{12}$. We distinguish the following cases:

Case 1: $z_{1}<\frac{1}{2}$ and $z_{2}<\frac{1}{2}$ (cf. case 1 in step 1): This occurs with probability $\frac{1}{4}$, and the outcome is $x_{1}=\min \left\{z_{1}, z_{2}\right\}$ with conditional expectation $\frac{1}{6}$ (cf. lemma 8.1 in appendix).

Case 2: $z_{1} \geq \frac{1}{2}$ and $z_{2} \geq \frac{1}{2}$ (cf. case 2 in step 1): This occurs with probability $\frac{1}{4}$, and the outcome is $x_{1}=\min \left\{z_{1}, z_{2}\right\}$ with conditional expectation $\frac{2}{3}$ (cf. lemma 8.1 in appendix).

Case 3: $z_{1}<\frac{1}{2} \leq z_{2}$ or $z_{2}<\frac{1}{2} \leq z_{1}$ (cf. case 3 in step 1): This occurs with probability $\frac{1}{2}$, and the outcome is $x_{2}=\max \left\{z_{1}, z_{2}\right\}$ with conditional expectation $\frac{3}{4}$.

Hence,

$$
U\left(\sigma^{1}, a\right)=\frac{1}{4} \cdot \frac{1}{6}+\frac{1}{4} \cdot \frac{2}{3}+\frac{1}{2} \cdot \frac{3}{4}=\frac{7}{12},
$$

completing the proof.

Remark: In the game $G_{2}$, player 1 in fact has many different optimal strategies, one of which is described in the theorem above. All optimal strategies coincide, up to a set of measure 
zero, with $\sigma^{1}$ in Cases 1 and 2 of Step 1, but may show different behavior in Case 3. The reason is that in Case 3 any behavior for player 1 is a best reply against $a=\frac{1}{2}$.

\section{Stationary Threshold Strategies for Player 2}

In this section, we identify the best strategy that player 2 has amongst all stationary threshold strategies. It turns out that this strategy is exactly the same as the one we found in Section 3. This means that player 2 , if he is restricted to stationary threshold strategies, will behave identically irrespective of whether player 1 chooses strategically or not.

Theorem 5.1. (Best stationary threshold strategy) Consider the game $G_{n}$. Player 2's best stationary threshold strategy is $a_{n}^{*}=\left(\frac{1}{n}\right)^{\frac{1}{n-1}}$, i.e. for any stationary threshold strategy a of player 2 we have $\psi^{2}\left(a_{n}^{*}\right) \geq \psi^{2}(a)$. Moreover, $a_{n}^{*}$ guarantees

$$
\psi^{2}\left(a_{n}^{*}\right)=\frac{1}{n+1}+a_{n}^{*}-\left(a_{n}^{*}\right)^{n}=\frac{1}{n+1}+\frac{n-1}{n} a_{n}^{*} .
$$

Proof. It is clear that $a=0$ or $a=1$ cannot be player 2's best stationary threshold strategy. Therefore, take an arbitrary $a \in(0,1)$. In order to find $\psi^{2}(a)$, we identify a best reply for player 1. In the following, let $x_{1}, \ldots, x_{n}$ be a permutation of $z_{1}, \ldots, z_{n}$ such that $x_{1} \leq x_{2} \leq \ldots \leq x_{n}$. We distinguish two cases:

Case 1: $x_{1} \leq \ldots \leq x_{n}<a$. This case occurs with probability $a^{n}$, and the outcome with best play by player 1 is $x_{1}$ (player 1 should keep $x_{1}$ for the last period when it has to be accepted). The conditional expectation of $x_{1}$ is

$$
\frac{n \cdot 0+a}{n+1}=\frac{a}{n+1}
$$

(cf. lemma 8.1 in appendix).

Case 2: $x_{1} \leq \ldots \leq x_{k}<a \leq x_{k+1} \leq \ldots \leq x_{n}$ with some $k \in\{0, \ldots, n-1\}$. This case occurs with probability

$$
\left(\begin{array}{l}
n \\
k
\end{array}\right) \cdot a^{k} \cdot(1-a)^{n-k},
$$

and the outcome with best play by player 1 is $x_{k+1}$ (all $x_{1}, \ldots, x_{k}$ are rejected by player 2 , and the lowest amount player 2 accepts is $x_{k+1}$ ). The conditional expectation of $x_{k+1}$ is

$$
\frac{(n-k) \cdot a+1}{n-k+1}
$$

(cf. lemma 8.1 in appendix). 
So the expected utility when player 1 uses a best reply $\sigma^{1}$ to $a$ is

$$
U\left(\sigma^{1}, a\right)=a^{n} \cdot \frac{a}{n+1}+\sum_{k=0}^{n-1}\left(\begin{array}{l}
n \\
k
\end{array}\right) \cdot a^{k} \cdot(1-a)^{n-k} \cdot \frac{(n-k) \cdot a+1}{n-k+1} .
$$

Somewhat surprisingly, Lemma 8.3 guarantees that the above expression reduces to

$$
U\left(\sigma^{1}, a\right)=\frac{1}{n+1}+a-a^{n}
$$

By taking derivatives, it easily follows that $U\left(\sigma^{1}, a\right)$ has a unique maximum at $a_{n}^{*}=\left(\frac{1}{n}\right)^{\frac{1}{n-1}}$, which is in $[0,1]$.

Remark: Lemma 8.2 shows that $a_{n}^{*}=\left(\frac{1}{n}\right)^{\frac{1}{n-1}}$ converges to 1 when $n$ tends to infinity. Note also that the probability of the maximal amount $\max \left\{z_{1}, \ldots, z_{n}\right\}$ being at least $a_{n}^{*}$ is exactly

$$
\alpha_{n}:=1-\left(a_{n}^{*}\right)^{n}=1-\frac{1}{n} a_{n}^{*} .
$$

Hence, if player 2 uses the stationary threshold strategy $a_{n}^{*}$, then the probability that player 2 eventually accepts an amount above the threshold $a_{n}^{*}$ is also exactly $\alpha_{n}$. Since $\alpha_{n}$ converges to 1 when $n$ tends to infinity, the strategy $a_{n}^{*}$ will accept an amount above $a_{n}^{*}$ with probability close to 1 , for large $n$. This, of course, also means that $\psi^{2}\left(a_{n}^{*}\right)$ converges to 1 , although this also follows directly from the expression for $\psi^{2}\left(a_{n}^{*}\right)$ in Theorem 5.1. The following table shows an approximation of $a_{n}^{*}$ and $\psi^{2}\left(a_{n}^{*}\right)$ for some values of $n$ :

\begin{tabular}{|c|c|c|c|c|c|c|c|c|}
\hline$n$ & 2 & 3 & 4 & 5 & 10 & 20 & 50 & 100 \\
\hline$a_{n}^{*}$ & $=0.5$ & $\approx 0.58$ & $\approx 0.63$ & $\approx 0.67$ & $\approx 0.77$ & $\approx 0.85$ & $\approx 0.92$ & $\approx 0.95$ \\
\hline$\psi^{2}\left(a_{n}^{*}\right)$ & $\approx 0.58$ & $\approx 0.63$ & $\approx 0.67$ & $\approx 0.70$ & $\approx 0.79$ & $\approx 0.86$ & $\approx 0.92$ & $\approx 0.95$ \\
\hline
\end{tabular}

Interestingly, $a_{n+1}^{*}=\left(\frac{1}{n+1}\right)^{\frac{1}{n}}$ is a very good approximation of $\psi^{2}\left(a_{n}^{*}\right)$ for all $n$, i.e. $a_{n+1}^{*} \geq$ $\psi^{2}\left(a_{n}^{*}\right)$ for all $n \geq 2$ and

$$
\max _{n \geq 2}\left(a_{n+1}^{*}-\psi^{2}\left(a_{n}^{*}\right)\right)=a_{3}^{*}-\psi^{2}\left(a_{2}^{*}\right) \approx 0.006 .
$$

Regarding the comparison between $\psi^{2}\left(a_{n}^{*}\right)$ and $\widetilde{v}_{n}$ (cf. Section 3), we remark that $\widetilde{v}_{n} \geq \psi^{2}\left(a_{n}^{*}\right)$ for all $n \geq 2$ and that

$$
\max _{n \geq 2}\left(\widetilde{v}_{n}-\psi^{2}\left(a_{n}^{*}\right)\right) \leq 0.08 .
$$

Since the value $v_{n}$ of the game $G_{n}$ satisfies $v_{n} \in\left[\psi^{2}\left(a_{n}^{*}\right), \widetilde{v}_{n}\right]$, the stationary threshold strategy $a_{n}^{*}$ is quite effective for all $n \geq 2$. 


\section{General Threshold Strategies for Player 2}

In this section, we examine general threshold strategies for player 2. First we show that we may restrict our investigation to threshold strategies $\sigma^{2}$ with the following property: if $\sigma^{2}$ prescribes threshold $b_{k}$ at period $k$, and the offered amount is below $b_{k}$ and gets rejected by $\sigma^{2}$, then the new threshold $b_{k+1}$ at period $k+1$ satisfies $b_{k} \geq b_{k+1}$. Thus, the thresholds are non-increasing during any play.

Theorem 6.1. (Thresholds are non-increasing) Consider an arbitrary threshold strategy $\sigma^{2}$ for player 2. Define another threshold strategy $\widetilde{\sigma}^{2}$ for player 2 as follows: At period 1, the strategy $\widetilde{\sigma}^{2}$ prescribes the same threshold as $\sigma^{2}$, i.e. $\widetilde{\sigma}^{2}(\emptyset):=\sigma^{2}(\emptyset)$, where $\emptyset$ denotes the empty history at period 1. At any period $k \geq 2$, if $\left(y_{1}, \ldots, y_{k-1}\right)$ denotes the sequence of past rejected amounts, then let

$$
\widetilde{\sigma}^{2}\left(y_{1}, \ldots, y_{k-1}\right):=\min _{\ell=1, \ldots, k} \sigma^{2}\left(y_{1}, \ldots, y_{\ell-1}\right) .
$$

Then, $\widetilde{\sigma}^{2}$ has the following properties:

1. The threshold strategy $\tilde{\sigma}^{2}$ is at least as good as $\sigma^{2}$, i.e. $\psi^{2}\left(\widetilde{\sigma}^{2}\right) \geq \psi^{2}\left(\sigma^{2}\right)$.

2. With respect to $\widetilde{\sigma}^{2}$, the thresholds are non-increasing during any play: at any period $k \geq 2$ and for any sequence $\left(y_{1}, \ldots, y_{k-1}\right)$ of past amounts that $\widetilde{\sigma}^{2}$ has rejected, we have

$$
\widetilde{\sigma}^{2}\left(y_{1}, \ldots, y_{k-2}\right) \geq \tilde{\sigma}^{2}\left(y_{1}, \ldots, y_{k-1}\right) .
$$

Proof. Property (2) is obvious, so we only have to show the property (1). Let $\tau^{1}$ be a pure best reply of player 1 to $\widetilde{\sigma}^{2}$. Take an arbitrary realization vector $\mathbf{z}=\left(z_{1}, \ldots, z_{n}\right)$, and suppose that $\tau^{1}$ prescribes the realizations in the order $y_{1}, \ldots, y_{n}$. Let $m$ denote the period at which $\widetilde{\sigma}^{2}$ accepts $y_{m}$. Thus, $y_{m}=U_{\mathbf{z}}\left(\tau^{1}, \widetilde{\sigma}^{2}\right)$ and $y_{m} \geq \widetilde{\sigma}^{2}\left(y_{1}, \ldots, y_{m-1}\right)$. If $m>1$, we may assume without loss of generality that $y_{m}<\widetilde{\sigma}^{2}\left(y_{1}, \ldots, y_{m-2}\right)$, because otherwise $\tau^{1}$ could just as well offer $y_{m}$ already at period $m-1$ since $\widetilde{\sigma}^{2}$ would accept it. This means

$$
\widetilde{\sigma}^{2}\left(y_{1}, \ldots, y_{m-1}\right) \leq y_{m}<\widetilde{\sigma}^{2}\left(y_{1}, \ldots, y_{m-2}\right),
$$

which implies

$$
\widetilde{\sigma}^{2}\left(y_{1}, \ldots, y_{m-1}\right)=\sigma^{2}\left(y_{1}, \ldots, y_{m-1}\right) .
$$

We now show that $U_{\mathbf{z}}\left(\tau^{1}, \sigma^{2}\right)=y_{m}$. Since the threshold prescribed by $\widetilde{\sigma}^{2}$ is never higher than the threshold prescribed by $\sigma^{2}$, it is clear that $\sigma^{2}$ also rejects $y_{1}, \ldots, y_{m-1}$ up to period $m-1$. But then, at period $m$, the strategy $\sigma^{2}$ also accepts $y_{m}$ in view of $(6.1)$. Thus, $U_{\mathbf{z}}\left(\tau^{1}, \sigma^{2}\right)=y_{m}$ indeed.

Therefore, $U_{\mathbf{z}}\left(\tau^{1}, \widetilde{\sigma}^{2}\right)=y_{m}=U_{\mathbf{z}}\left(\tau^{1}, \sigma^{2}\right)$, which implies

$$
\psi^{2}\left(\widetilde{\sigma}^{2}\right)=U\left(\tau^{1}, \widetilde{\sigma}^{2}\right)=U\left(\tau^{1}, \sigma^{2}\right) \geq \psi^{2}\left(\sigma^{2}\right),
$$

completing the proof of property (1). 
Corollary 6.2. Let $\mathbf{a}=\left(a_{1}, \ldots, a_{n-1}\right)$ be a Markov threshold strategy for player 2. Then, there exists a Markov threshold strategy $\mathbf{b}=\left(b_{1}, \ldots, b_{n-1}\right)$ for player 2 such that $b_{k} \geq b_{k+1}$ for all $k \in\{1, \ldots, n-2\}$ and for which $\psi^{2}(\mathbf{b}) \geq \psi^{2}(\mathbf{a})$. Consequently, a best strategy amongst the Markov threshold strategies for player 2 consists of a non-increasing sequence of thresholds. ${ }^{6}$

The above corollary can also be shown in a more direct way. First, one can prove the following statement about transpositions of neighboring thresholds. Let $\mathbf{a}=\left(a_{1}, \ldots, a_{n-1}\right)$ be a Markov threshold strategy for which $a_{k}<a_{k+1}$ holds for some $k \in\{1, \ldots, n-2\}$. Let $\mathbf{b}=\left(b_{1}, \ldots, b_{n-1}\right)$ denote the Markov threshold strategy obtained by $b_{k}=a_{k+1}$ and $b_{k+1}=a_{k}$, while $b_{m}=a_{m}$ for all $m \in\{1, \ldots, k-1, k+2, \ldots, n-1\}$. Then, it can be shown that $\psi^{2}(\mathbf{b}) \geq \psi^{2}(\mathbf{a})$. Given this result, the corollary above follows by the well known theorem in algebra that any permutation can be written as a product of transpositions of two neighboring elements.

Theorem 6.3. (Stationary threshold strategies are not optimal) When $n \geq 3$, player 2 has a threshold strategy which is strictly better than all stationary threshold strategies.

Proof. Let $n \geq 3$. In view of Theorem 5.1, it suffices to construct a threshold strategy for player 2 which is strictly better than the stationary threshold strategy $a=\left(\frac{1}{n}\right)^{\frac{1}{n-1}}$. Let $b \in(0, a)$ be arbitrary. Let $\sigma_{b}^{2}$ be the threshold strategy for player 2 which prescribes threshold $a$ at every period, except in the following case: at period $n-1$ (which is the last period when player 2 has a choice), if all $n-2$ previously rejected amounts are in the interval $[b, a)$, then use threshold $b$ at period $n-1$.

Now we show that $\sigma_{b}^{2}$ is strictly better than strategy $a$ for player 2 , if $b$ is sufficiently close to threshold $a$. Let $\left(z_{1}, \ldots, z_{n}\right)$ denote the realizations of the amounts. We distinguish the following cases:

Case 1: Less than $n-2$ amounts in $z_{1}, \ldots, z_{n}$ are in the interval $[b, a)$. In this case, $\sigma_{b}^{2}$ is the same as $a$.

Case 2: Precisely $n-2$ amounts in $z_{1}, \ldots, z_{n}$ are in the interval $[b, a)$. In this case, $\sigma_{b}^{2}$ can only prescribe threshold $b$ at period $n-1$ if player 1 offers precisely these $n-2$ amounts in $[b, a)$ at periods up to $n-2$. But then, for periods $n-1$ and $n$, there is no amount left in $[b, a)$. Hence, in this case, $\sigma_{b}^{2}$ is equally good as $a$.

Case 3: Precisely $n-1$ amounts in $z_{1}, \ldots, z_{n}$ are in the interval $[b, a)$. Let $\underline{z}$ denote the minimum of these $n-1$ amounts in $[b, a)$ and let $w$ denote the amount outside $[b, a)$. We show that, with best play by player 1 , strategy $\sigma_{b}^{2}$ yields outcome $\underline{z}$ and $a$ yields outcome $w$.

Case 3i: $w<b$. In this case, with best play by player 1 , strategy $\sigma_{b}^{2}$ yields outcome $\underline{z}$. Indeed, if player 1 offers all amounts in $[b, a)$ except $\underline{z}$ at periods up to $n-2$ and offers $\underline{z}$ at period $n-1$, strategy $\sigma_{b}^{2}$ will accept $\underline{z}$ as $\underline{z} \geq b$. On the other hand, $w$ cannot be the outcome for the following reason. Player 2 would only accept $w$ at the last period, as $w<b$. Thus, to

\footnotetext{
${ }^{6}$ It can be shown, also by the argument after the corollary, that all best Markov threshold strategies have non-increasing thresholds.
} 
achieve $w$, player 1 would have to offer the $n-1$ amounts in $[b, a)$ at periods up to $n-1$. But then, $\sigma_{b}^{2}$ accepts the amount at period $n-1$. The strategy $a$, on the other hand, yields $w$ as outcome, because player 1 can reserve $w$ for the last period, when it has to be accepted.

Case 3ii: $w \geq a$. In this case, with best play by player 1 , strategy $\sigma_{b}^{2}$ yields outcome $\underline{z}$. Indeed, if player 1 offers all amounts in $[b, a)$ except $\underline{z}$ at periods up to $n-2$ and offers $\underline{z}$ at period $n-1$, strategy $\sigma_{b}^{2}$ will accept $\underline{z}$ as $\underline{z} \geq b$. The strategy $a$, on the other hand, yields $w$, as $a$ rejects all other amounts.

Case 4: All $n$ amounts in $z_{1}, \ldots, z_{n}$ are in the interval $[b, a)$. In this case, with best play by player 1 , strategy $\sigma_{b}^{2}$ yields $\min \left\{z_{1}, \ldots, z_{n}\right\}$ as outcome, since player 1 can offer the minimal amount at period $n-1$. The strategy $a$ also yields $\min \left\{z_{1}, \ldots, z_{n}\right\}$ as outcome, because player 1 can reserve the minimal amount for the last period, when it has to be accepted. Hence, in this case, $\sigma_{b}^{2}$ is equally good as $a$.

In conclusion, $\sigma_{b}^{2}$ is equally good as $a$ in all cases except for case 3 . On condition that case 3 occurs, we obtain the following. Recall that, with best play by player 1 , strategy $\sigma_{b}^{2}$ yields outcome $\underline{z}$ and strategy $a$ yields outcome $w$. Let $E_{\underline{z}}(b)$ denote the conditional expected value of $\underline{z}$ and let $E_{w}(b)$ denote the conditional expected value of $w$ in case 3 . In order to show that strategy $\sigma_{b}^{2}$ is strictly better than strategy $a$, for $b$ sufficiently close to $a$, we need to show that

$$
\lim _{b \uparrow a} E_{\underline{z}}(b)>\lim _{b \uparrow a} E_{w}(b) .
$$

Since $\underline{z} \in[b, a)$ in case 3 , we have

$$
\lim _{b \uparrow a} E_{\underline{z}}(b)=a .
$$

We calculate $\lim _{b \uparrow a} E_{w}(b)$ in the following way. Subcase 3i appears with conditional probability

$$
p_{b}=\frac{b}{b+(1-a)},
$$

and the conditional expectation of $w$ is $\frac{b}{2}$. Subcase 3ii appears with conditional probability $1-p_{b}$, and the conditional expectation of $w$ is

$$
\frac{a+1}{2} \text {. }
$$

Hence,

$$
E_{w}(b)=p_{b} \cdot \frac{b}{2}+\left(1-p_{b}\right) \cdot \frac{a+1}{2} .
$$

By taking the limit, we obtain

$$
\lim _{b \uparrow a} E_{w}(b)=\frac{a^{2}}{2}+\frac{1-a^{2}}{2}=\frac{1}{2} .
$$


In conclusion,

$$
\lim _{b \uparrow a} E_{\underline{z}}(b)=a>\frac{1}{2}=\lim _{b \uparrow a} E_{w}(b),
$$

which completes the proof.

Theorem 6.4. (Markov threshold strategies are not optimal) When $n \geq 3$, player 2 has a threshold strategy which is strictly better than all Markov threshold strategies.

Proof. Let $n \geq 3$. Take a best Markov threshold strategy $\mathbf{a}=\left(a_{1}, \ldots, a_{n-1}\right)$ for player 2 . We construct a threshold strategy $\sigma^{2}$ for player 2 which is strictly better than a. In view of Corollary 6.2 and Theorem 6.3 , we may assume that $a_{1} \geq \ldots \geq a_{k}>a_{k+1} \geq \ldots \geq a_{n-1}$. It can easily be verified that $a_{1}=1$ or $a_{n-1}=0$ can never yield a best strategy amongst all Markov threshold strategies, and hence we assume that $a_{1}<1$ and $a_{n-1}>0$.

Consider the threshold strategy $\sigma^{2}$ for player 2 which prescribes the same thresholds as a except in the following case: at period $k+1$, if the rejected amount at period $k$ was in the interval $\left[0, a_{k+1}\right)$, then use threshold $a_{k}$ at period $k+1$. We show that, with best play by player 1 , the strategy $\sigma^{2}$ is strictly better than a for player 2 .

Step 1: We show that, for every vector of realizations $\mathbf{z}=\left(z_{1}, \ldots, z_{n}\right)$, the strategy $\sigma^{2}$ is at least as good as a for player 2 .

Let $\mathbf{z}=\left(z_{1}, \ldots, z_{n}\right)$ be a vector of realizations. Let $\sigma^{1}$ denote a pure best reply for player 1 to $\sigma^{2}$, and suppose that $\sigma^{1}$ offers these amounts in the order $\left(y_{1}, \ldots, y_{n}\right)$. Let $\tau^{1}$ be the strategy for player 1 which also uses the order $\left(y_{1}, \ldots, y_{n}\right)$, except in the following case: if $y_{k}<a_{k+1}$ and $y_{k+1} \in\left[a_{k+1}, a_{k}\right)$, then use the order

$$
\left(y_{1}, \ldots, y_{k-1}, y_{k+1}, y_{k}, y_{k+2}, \ldots, y_{n}\right) \text {. }
$$

We show that $U_{\mathbf{z}}\left(\sigma^{1}, \sigma^{2}\right)=U_{\mathbf{z}}\left(\tau^{1}, \mathbf{a}\right)$, which will imply that $\psi^{2}\left(\sigma^{2}\right) \geq \psi^{2}(\mathbf{a})$. We may assume that all $y_{1}<a_{1}, \ldots, y_{k-1}<a_{k-1}$ (i.e. they are all rejected) and that $y_{k}<a_{k+1}$, otherwise $\left(\sigma^{1}, \sigma^{2}\right)$ and $\left(\tau^{1}, \mathbf{a}\right)$ lead to the same outcome. We distinguish the following cases:

Case 1: If $y_{k+1} \geq a_{k}$. In this case, $U_{\mathbf{z}}\left(\sigma^{1}, \sigma^{2}\right)=U_{\mathbf{z}}\left(\tau^{1}, \mathbf{a}\right)=y_{k+1}$.

Case 2: If $y_{k+1} \in\left[a_{k+1}, a_{k}\right)$. In this case, $\sigma^{2}$ will use threshold $a_{k}$ at period $k+1$. Thus, both $\left(\sigma^{1}, \sigma^{2}\right)$ and $\left(\tau^{1}, \mathbf{a}\right)$ lead to rejections at periods $k$ and $k+1$, and hence $U_{\mathbf{z}}\left(\sigma^{1}, \sigma^{2}\right)=U_{\mathbf{z}}\left(\tau^{1}, \mathbf{a}\right)$.

Case 3. If $y_{k+1}<a_{k+1}$. Also in this case, both $\left(\sigma^{1}, \sigma^{2}\right)$ and $\left(\tau^{1}, \mathbf{a}\right)$ lead to rejections at periods $k$ and $k+1$, and hence $U_{\mathbf{z}}\left(\sigma^{1}, \sigma^{2}\right)=U_{\mathbf{z}}\left(\tau^{1}, \mathbf{a}\right)$.

Step 2: We show that there exists a set $W$ of realization vectors such that $W$ has a positive probability and that, for every realization vector in $W$, the strategy $\sigma^{2}$ is strictly better than a for player 2 .

Let $W$ denote the set of realization vectors in which exactly $k$ amounts are in the interval $\left[0, a_{n-1}\right)$, exactly 1 amount is in the interval $\left[a_{k+1}, a_{k}\right)$, and exactly $n-k-1$ amounts are in 
the interval $\left[a_{1}, 1\right]$. Of course, $W$ has a positive probability, since $a_{1}<1$ and $a_{n-1}>0$. Take an arbitrary realization vector in $W$. Notice that, against $\mathbf{a}$, it is a best reply for player 1 to offer the $k$ amounts in $\left[0, a_{n-1}\right)$ at periods up to $k$, which all get rejected, and then at period $k+1$ to offer the amount in $\left[a_{k+1}, a_{k}\right)$, which is accepted. This does not work against $\sigma^{2}$, since in this case, $\sigma^{2}$ uses threshold $a_{k}$ at period $k+1$. It is easy to see that $\sigma^{2}$ leads to an outcome in $\left[a_{1}, 1\right]$, regardless player 1's strategy.

Two important questions arise:

Question (1): Which are the best threshold strategies for player 2?

Question (2): Does player 2 have better strategies than threshold strategies, i.e. are there optimal strategies in threshold strategies for player 2 ?

Question (1) is already challenging for $n=3$. The best threshold strategy for player 2 that we could find is the following. Let $a \in(0,1)$ and $b \in(0, a)$ be arbitrary. Let $\sigma_{a b}^{2}$ be the threshold strategy for player 2 which prescribes threshold $a$ at period 1 , and prescribes threshold $a$ at period 2 if the rejected amount was in interval $[0, b)$ and threshold $b$ at period 2 if the rejected amount was in interval $[b, a)$. (This is very similar to strategy $\sigma_{b}^{2}$ in the proof of Theorem 6.3 , with the only difference being that $a$ here is also a variable.) Let $a^{*}$ and $b^{*}$ denote optimal values for $a$ and $b$. According to a numerical approximation by the program package Mathematica, $a^{*} \approx 0.5838$ and $b^{*} \approx 0.4975$. The strategy $\sigma_{a^{*} b^{*}}^{2}$ guarantees $\psi^{2}\left(\sigma_{a^{*} b^{*}}^{2}\right) \approx 0.6354$ (the best stationary threshold strategy found in Theorem 5.1 yields 0.6349 , which is just "slightly" less). We did not manage to find an improvement on $\sigma_{a^{*} b^{*}}^{2}$ for player 2. It seems natural to try to find an improvement by splitting $[0, a)$ into more than 2 subintervals. Thus, instead of $a$ and $b$ only, now player 2 can choose $a, b, c, d \in(0,1)$, with $d \leq c \leq b \leq a$, and a map $\alpha:\{a, b, c, d\} \rightarrow\{a, b, c, d\}$. Then, player 2 will use the following threshold strategy: At period 1, use threshold $a$. If the offered amount $y^{1}$ is rejected, then the new threshold at period 2 will depend on which of the subintervals $[0, d),[d, c),[c, b),[b, a)$ contains $y^{1}$. More precisely, if $y^{1}$ is contained in subinterval $[u, w)$, then the new threshold is $\alpha(w)$. With the help of the program package Mathematica, with a numerical precision of $10^{-10}$, we found the surprising conclusion that $\sigma_{a^{*} b^{*}}^{2}$ is still a best amongst these strategies, i.e. one of the optimal choices is $a=a^{*}, b=c=d=b^{*}$ and $\alpha(a)=b$, $\alpha(b)=a$. We do not see now how one could improve upon $\sigma_{a^{*} b^{*}}^{2}$. It is not even really clear to us why $\sigma_{a^{*} b^{*}}^{2}$ is so effective, although the proof of Theorem 6.3 provides some ideas.

We do not know the answer to Question (2). We only know that an optimal threshold strategy exists for player 2 in $G_{2}$, cf. Section 4 . 


\section{Concluding Remarks}

\subsection{Are Markov threshold strategies really better for player 2 than stationary threshold strategies?}

Notice that corollary 6.2 does not exclude the possibility that the best Markov threshold strategy is the stationary threshold strategy which we derived in Section 5. In fact, we conjecture that this holds true for all $n$. For $n=1$ and $n=2$, this is trivial, since player 2 uses at most one threshold. For $n=3$, we verified this conjecture in the following way. For an arbitrary Markov threshold strategy $\sigma^{2}=\left(a_{1}, a_{2}\right)$ for player 2 with non-increasing thresholds $a_{1} \geq a_{2}$, we determined a best response for player 1 , and calculated the corresponding expected outcome, as a function of $a_{1}$ and $a_{2}$. Then, we checked that this expected outcome is indeed maximal when $a_{1}=a_{2}=\left(\frac{1}{3}\right)^{\frac{1}{2}}$. For $n=4$ and $n=5$, the program package Mathematica confirms this conjecture (with a numerical precision of $10^{-5}$ ). For a general $n$, it is difficult to prove this conjecture. First of all, the proof we used for $n=3$ produces huge polynomials when $n$ is large. It seems more natural to try a proof based on induction. Perhaps, the best candidate is to try to show the following statement: if $\mathbf{a}=\left(a_{1}, a_{2}, \ldots, a_{n-1}\right)$ is a Markov threshold strategy for player 2 with thresholds

$$
a_{1} \geq \ldots \geq a_{k}>a_{k+1}=\ldots=a_{n-1},
$$

then there is a strictly better Markov threshold strategy of the form $\widetilde{\mathbf{a}}=\left(a_{1}, \ldots, a_{k}, b, \ldots, b\right)$ with an appropriate threshold $b \in\left[a_{k+1}, a_{k}\right]$.

\subsection{Effective strategies for player 1}

In this section, we focus on player 1. The main difference is that here we have to deal with more complex strategies, as player 1 can base his decisions on the realization vector. In general for $n \geq 3$, we have not been able to find an optimal strategy for player 1 . Nevertheless, we provide some insight, and present effective strategies for player 1 with a simple structure (although just offering amount $z_{k}$ at period $k$ for every $k$, as in Section 3, is already quite effective, especially for large $n$ ).

Recall that, for any $n \geq 3$, the value $v_{n}$ of the game satisfies $v_{n} \in\left[\psi^{2}\left(a_{n}^{*}\right), \widetilde{v}_{n}\right]$, where $\psi^{2}\left(a_{n}^{*}\right)$ and $\widetilde{v}_{n}$ are determined in Sections 5 and 3, respectively. We reiterate the approximations:

\begin{tabular}{|c|c|c|c|c|c|c|c|c|}
\hline$n$ & 2 & 3 & 4 & 5 & 10 & 20 & 50 & 100 \\
\hline$\widetilde{v}_{n}$ & $\approx 0.63$ & $\approx 0.70$ & $\approx 0.74$ & $\approx 0.78$ & $\approx 0.86$ & $\approx 0.92$ & $\approx 0.96$ & $\approx 0.98$ \\
\hline$\psi^{2}\left(a_{n}^{*}\right)$ & $\approx 0.58$ & $\approx 0.63$ & $\approx 0.67$ & $\approx 0.70$ & $\approx 0.79$ & $\approx 0.86$ & $\approx 0.92$ & $\approx 0.95$ \\
\hline
\end{tabular}

As pointed out in (5.1),

$$
\max _{n \geq 2}\left(\widetilde{v}_{n}-\psi^{2}\left(a_{n}^{*}\right)\right) \leq 0.08
$$


which means that the strategy for player 1 in which he offers every realization $z_{k}$ at period $k$, is already 0.08-optimal. Nevertheless, player 1 can do better than $\widetilde{v}_{n}$. Below we provide some possible improvements.

Improvement 1: Based on the results for $n=2$ in Section 4, there is one simple improvement for player 1 for all $n \geq 3$. Let $\xi_{n}^{1}$ be the strategy for player 1 which, for any realization vector $\mathbf{z}=\left(z_{1}, \ldots, z_{n}\right)$, prescribes the following: At any period $k \leq n-2$, offer $z_{k}$ to player 2 . At the last two periods, i.e. at periods $n-1$ and $n$, play the strategy found in Section 4 with the two remaining amounts $z_{n-1}$ and $z_{n}$. Now we determine $u_{n}:=\psi^{1}\left(\xi_{n}^{1}\right)$. We have $u_{2}=v_{2}=\frac{7}{12}$ according to Theorem 4.1. We proceed by calculating $u_{3}$. Just as in the proof of Theorem 3.1, player 2 should accept the offered amount $y$ at period 1 if $y \geq v_{2}=\frac{7}{12}$, and reject it otherwise. Thus, player 2 accepts $y$ with probability $1-v_{2}$ and with conditional expected amount $\frac{1}{2}\left(v_{2}+1\right)$, and rejects with probability $v_{2}$. Therefore,

$$
u_{3}=\left(1-v_{2}\right) \cdot \frac{1}{2}\left(v_{2}+1\right)+v_{2} \cdot v_{2}=\frac{1}{2}+\frac{1}{2}\left(v_{2}\right)^{2} .
$$

Using this argument inductively, we obtain for all $n \geq 3$ that

$$
u_{n}=\frac{1}{2}+\frac{1}{2}\left(u_{n-1}\right)^{2} .
$$

We obtained a similar recursion for $\widetilde{v}_{n}$ in Theorem 3.1. As $\widetilde{v}_{2}=\frac{5}{8}>\frac{7}{12}=u_{2}$, we may conclude $\widetilde{v}_{n}>u_{n}$ for all $n \geq 2$. The following table provides an approximation of $u_{n}$ for some values of $n:$

\begin{tabular}{|c|c|c|c|c|c|c|c|c|}
\hline$n$ & 2 & 3 & 4 & 5 & 10 & 20 & 50 & 100 \\
\hline$\psi^{1}\left(\xi_{n}^{1}\right)=u_{n}$ & $\approx 0.58$ & $\approx 0.67$ & $\approx 0.72$ & $\approx 0.76$ & $\approx 0.86$ & $\approx 0.92$ & $\approx 0.96$ & $\approx 0.98$ \\
\hline
\end{tabular}

Note that

$$
\max _{n \geq 2}\left(\psi^{1}\left(\xi_{n}^{1}\right)-\psi^{2}\left(a_{n}^{*}\right)\right) \leq 0.07
$$

which means that $\xi_{n}^{1}$ is 0.07 -optimal for player 1 .

Improvement 2: Recall from Section 4 that, for $n=2$, it was optimal for player 1 to choose the amount closer to $\frac{1}{2}$. We now try to generalize this strategy for $n=3$. Take some $w \in\left[\frac{1}{2}, 1\right]$. Let $\sigma_{w}^{1}$ be the strategy for player 1 which, for realizations $z_{1}, z_{2}, z_{3}$, prescribes the following: At period 1, player 1 should offer the amount closest to $w$. If this amount is rejected, then at period 2 , player 1 should offer the amount amongst the two remaining amounts which is closer to $w$.

Suppose that, against $\sigma_{w}^{1}$, player 2 uses a pure threshold strategy $\sigma_{w}^{2}$. (One can show, based on the discussion below, that player 2 has a best reply in threshold strategies.) Let $a_{w}$ denote the threshold prescribed by $\sigma_{w}^{2}$ at period 1 . If $\sigma_{w}^{2}$ rejects amount $y_{1} \in\left[0, a_{w}\right)$ at period 1 , it is 
relatively easy to determine the best threshold for period 2. Let $y_{2}$ and $y_{3}$ denote the amounts chosen by $\sigma_{w}^{1}$ for periods 2 and 3. We distinguish the following cases.

Case 1: If $y_{2} \geq w$. In this case, since $y_{3}$ is not closer to $w$ than $y_{2}$, we have either $y_{3} \in\left[y_{2}, 1\right]$ or $y_{3} \in\left[0,2 w-y_{2}\right]$. This gives a conditional expectation of $y_{3}$ equal to

$$
d_{3}=\frac{\left(1-y_{2}\right) \cdot \frac{y_{2}+1}{2}+\left(2 w-y_{2}\right) \cdot \frac{2 w-y_{2}}{2}}{\left(1-y_{2}\right)+\left(2 w-y_{2}\right)} .
$$

As $w \geq \frac{1}{2}$, we have $1-y_{2} \leq 2 w-y_{2}$. Moreover, $y_{2} \geq w$ implies that

$$
\frac{y_{2}+1}{2} \geq \frac{2 w-y_{2}}{2} \text {. }
$$

Hence,

$$
d_{3} \leq \frac{1}{2}\left(\frac{y_{2}+1}{2}+\frac{2 w-y_{2}}{2}\right)=\frac{1}{4}(2 w+1) \leq w \leq y_{2} .
$$

This means that player 2 should accept $y_{2}$.

Case 2: If $y_{2}<w$. In this case, since $y_{3}$ is not closer to $w$ than $y_{2}$, we have either $y_{3} \in\left[0, y_{2}\right]$ or $y_{3} \in\left[2 w-y_{2}, 1\right]$ (with the latter interval being empty when $2 w-y_{2}>1$ ).

Case 2.1: If $2 w-y_{2}>1$. In this case, $y_{3} \in\left[0, y_{2}\right]$ and player 2 should accept $y_{2}$.

Case 2.2: If $2 w-y_{2} \leq 1$. In this case, the conditional expectation of $y_{3}$ is equal to

$$
d_{3}=\frac{y_{2} \cdot \frac{y_{2}}{2}+\left(1-2 w+y_{2}\right) \cdot \frac{2 w-y_{2}+1}{2}}{y_{2}+\left(1-2 w+y_{2}\right)} .
$$

Here, player 2 should accept $y_{2}$ exactly when $y_{2} \geq d_{3}$, which is a quadratic inequality.

By backwards induction, it would be possible to calculate the best threshold $a_{w}$ for period 1. We have found the following, with the help of a simulation with the program package Mathematica:

1. Regarding $\sigma_{w}^{1}$ : The best value of $w$ for player 1 is about $w=0.59$.

2. Regarding $\sigma_{w}^{2}$ : Against $\sigma_{0.59}^{1}$, the best value of threshold $a_{0.59}$ is about $a_{0.59}=0.59$. (This implies for case 2.2 , in view of (7.2), that player 2 should accept $y_{2}$ exactly when $y_{2} \leq 0.21$ or $y_{2} \geq 0.47$, approximately.)

3. Regarding the expected outcome: we have $\psi^{1}\left(\sigma_{0.59}^{1}\right) \approx U\left(\sigma_{0.59}^{1}, \sigma_{0.59}^{2}\right) \approx 0.639$.

This numerical simulation indicates that the strategy $\sigma_{0.59}^{1}$ is very effective, as $v_{3} \geq \psi^{2}\left(a_{3}^{*}\right) \approx$ 0.6349 (or even $v_{3} \geq \psi^{2}\left(\sigma_{a^{*} b^{*}}^{2}\right) \approx 0.6354$ where $\sigma_{a^{*} b^{*}}^{2}$ is the strategy given at the end of Section $6)$.

This also yields a possible improvement for player 1 for all $n \geq 4$, just as above. Let $\tau_{n}^{1}$ be the strategy for player 1 which, for any realization vector $\mathbf{z}=\left(z_{1}, \ldots, z_{n}\right)$, prescribes the following: At any period $k \leq n-3$, offer amount $z_{k}$ to player 2 . At the last three periods, play the strategy $\sigma_{0.59}^{1}$ with amounts $z_{n-2}, z_{n-1}$ and $z_{n}$. With a similar calculation as before we find the following approximate values of $\psi^{1}\left(\tau_{n}^{1}\right)$ for different values values of $n$ : 


\begin{tabular}{|c|c|c|c|c|c|c|c|}
\hline$n$ & 3 & 4 & 5 & 10 & 20 & 50 & 100 \\
\hline$\psi^{1}\left(\tau_{n}^{1}\right)$ & $\approx 0.64$ & $\approx 0.70$ & $\approx 0.75$ & $\approx 0.85$ & $\approx 0.92$ & $\approx 0.96$ & $\approx 0.98$ \\
\hline
\end{tabular}

with

$$
\max _{n \geq 2}\left(\psi^{1}\left(\tau_{n}^{1}\right)-\psi^{2}\left(a_{n}^{*}\right)\right) \leq 0.065 .
$$

We do not know whether, for $n>3$, a strategy similar to $\sigma_{w}^{1}$ would be effective for player 1 .

\subsection{The value $v_{n}$ and optimal strategies}

Except for $n=2$ in Section 4, we have not been able to determine the exact value $v_{n}$ of the game $G_{n}$ and to find optimal strategies for the players. Nevertheless, we know that $v_{2}=\frac{7}{12}$ and that $v_{n}$ is strictly increasing in $n$ and converges to 1 as $n$ tends to infinity. For an estimation of $v_{n}$, we may use the interval $I_{n}=\left[\psi^{2}\left(a_{n}^{*}\right), \psi^{1}\left(\xi_{n}^{1}\right)\right]$. The length of $I_{n}$ is at most 0.07 , in view of (7.1), and converges to 0 as $n$ tends to infinity. Consequently, the stationary threshold strategy $a_{n}^{*}$ is 0.07 -optimal for player 2 and the strategy $\xi_{n}^{1}$ is also 0.07 -optimal for player 1 . Note that, at the end of Sections 6 and 7.2, we provided possible improvements on these strategies (i.e. $\sigma_{a^{*} b^{*}}^{2}$ for $n=3$ and $\tau_{n}^{1}$ for $n \geq 3$ ), which also yield in turn a better estimate for the value $v_{n}$. We remark that, when $n=\infty$ (i.e. the case of countably infinite random variables), the value equals $v_{\infty}=1$ and any strategy of player 1 is optimal, whereas player 2 has only near-optimal strategies.

\section{Appendix}

Lemma 8.1. Let $W_{1}, \ldots, W_{m}$ denote independent random variables, each of which having a uniform distribution on some interval $[c, d]$. Then

$$
\mathbb{E}\left(\min \left\{W_{1}, \ldots, W_{m}\right\}\right)=\frac{m \cdot c+d}{m+1}
$$

and

$$
\mathbb{E}\left(\max \left\{W_{1}, \ldots, W_{m}\right\}\right)=\frac{c+m \cdot d}{m+1} .
$$

Proof. Let $W^{*}=\min \left\{W_{1}, \ldots, W_{m}\right\}$. We will show for the interval $[c, d]=[0,1]$ that

$$
\mathbb{E}\left(W^{*}\right)=\frac{1}{m+1} .
$$

Since $\max \left\{W_{1}, \ldots, W_{m}\right\}=1-\min \left\{1-W_{1}, \ldots, 1-W_{m}\right\}$, we obtain part 2 for interval $[0,1]$. It is easy to check, by using the linearity of the expectation, that the results hold for a general interval $[c, d]$ as well. 
So, take $[c, d]=[0,1]$. Let $W$ denote a random variable having a uniform distribution on interval $[0,1]$. Then, its density $f_{W}(t)$ is given by $f_{W}(t)=1$ for $t \in[0,1]$ and $f_{W}(t)=0$ otherwise. Also, its cumulative distribution $F_{W}(t)=\mathbb{P}(W \leq t)$ is given by $F_{W}(t)=0$ if $t<0$, and $F_{W}(t)=t$ if $t \in[0,1]$ and $F_{W}(t)=1$ if $t>1$.

As for $W^{*}$, we clearly have $F_{W^{*}}(t)=0$ if $t<0$ and $F_{W^{*}}(t)=1$ if $t>1$, whereas for $t \in[0,1]$

$$
\begin{aligned}
F_{W^{*}}(t) & =\mathbb{P}\left(W^{*} \leq t\right)=1-\mathbb{P}\left(W^{*}>t\right)=1-\mathbb{P}\left(\left\{W_{i}>t \forall i \in\{1, \ldots, m\}\right\}\right) \\
& =1-(\mathbb{P}(W>t))^{m}=1-\left(1-F_{W}(t)\right)^{m}=1-(1-t)^{m} .
\end{aligned}
$$

Hence, $f_{W^{*}}(t)=0$ if $t<0$ or $t>1$, while for $t \in[0,1]$

$$
f_{W^{*}}(t)=\frac{d}{d t} F_{W^{*}}(t)=m \cdot(1-t)^{m-1} .
$$

So,

$$
\begin{aligned}
\mathbb{E}\left(W^{*}\right) & =\int_{-\infty}^{\infty} t \cdot f_{W^{*}}(t) d t=\int_{0}^{1} t \cdot m \cdot(1-t)^{m-1} d t \\
& =m \cdot \int_{0}^{1}(1-u) \cdot u^{m-1} d u=m \cdot\left[\frac{1}{m} u^{m}-\frac{1}{m+1} u^{m+1}\right]_{u=0}^{u=1} \\
& =m \cdot\left[\frac{1}{m}-\frac{1}{m+1}\right]=\frac{1}{m+1},
\end{aligned}
$$

where we used the substitution $u=1-t$.

Lemma 8.2. $\lim _{n \rightarrow \infty}\left(\frac{1}{n}\right)^{\frac{1}{n-1}}=1$.

Proof. By using the rule of L'Hospital, we have

$$
\lim _{n \rightarrow \infty} \frac{\ln (n)}{n-1}=\lim _{n \rightarrow \infty} \frac{1}{n}=0 .
$$

Hence,

$$
\lim _{n \rightarrow \infty}\left(\frac{1}{n}\right)^{\frac{1}{n-1}}=\lim _{n \rightarrow \infty} n^{-\frac{1}{n-1}}=\lim _{n \rightarrow \infty} e^{\ln \left(n^{-\frac{1}{n-1}}\right)}=\lim _{n \rightarrow \infty} e^{-\frac{1}{n-1} \cdot \ln (n)}=e^{0}=1 .
$$

Lemma 8.3. For every $a \in \mathbb{R}-\{0,1\}$ and every $n \in \mathbb{N}$ it holds that

$$
\frac{a^{n+1}}{n+1}+\sum_{k=0}^{n-1}\left(\begin{array}{l}
n \\
k
\end{array}\right) \cdot a^{k} \cdot(1-a)^{n-k} \cdot \frac{(n-k) \cdot a+1}{n-k+1}=\frac{1}{n+1}+a-a^{n}
$$


Proof. Let $D_{n}$ be equal to the lefthandside of (8.1). Then,

$$
\begin{aligned}
D_{n} & =\frac{a^{n+1}}{n+1}+\sum_{k=0}^{n-1} \frac{n !}{k ! \cdot(n-k+1) !} \cdot a^{k} \cdot(1-a)^{n-k} \cdot((n-k+1) \cdot a+(1-a)) \\
& =\frac{a^{n+1}}{n+1}+\sum_{k=0}^{n+1} \frac{n !}{k ! \cdot(n-k+1) !} \cdot a^{k} \cdot(1-a)^{n-k} \cdot((n-k+1) \cdot a+(1-a))-a^{n}-\frac{a^{n+1}}{n+1} \\
& =\sum_{k=0}^{n+1} \frac{n !}{k ! \cdot(n-k+1) !} \cdot a^{k} \cdot(1-a)^{n-k} \cdot((n-k+1) \cdot a+(1-a))-a^{n} \\
& =\sum_{k=0}^{n+1} \frac{n ! \cdot(n+1-k)}{k ! \cdot(n+1-k) !} \cdot a^{k+1} \cdot(1-a)^{n-k}+\sum_{k=0}^{n+1} \frac{n !}{k ! \cdot(n-k+1) !} \cdot a^{k} \cdot(1-a)^{n+1-k}-a^{n} .
\end{aligned}
$$

Notice that

$$
\begin{aligned}
\sum_{k=0}^{n+1} \frac{n ! \cdot(n+1-k)}{k ! \cdot(n+1-k) !} \cdot a^{k+1} \cdot(1-a)^{n-k} & =a \cdot \sum_{k=0}^{n} \frac{n !}{k ! \cdot(n-k) !} \cdot a^{k} \cdot(1-a)^{n-k} \\
& =a \cdot(a+(1-a))^{n}=a
\end{aligned}
$$

and

$$
\begin{aligned}
\sum_{k=0}^{n+1} \frac{n !}{k ! \cdot(n-k+1) !} \cdot a^{k} \cdot(1-a)^{n+1-k} & =\frac{1}{n+1} \sum_{k=0}^{n+1} \frac{(n+1) !}{k ! \cdot(n+1-k) !} \cdot a^{k} \cdot(1-a)^{n+1-k} \\
& =\frac{1}{n+1}(a+(1-a))^{n+1}=\frac{1}{n+1} .
\end{aligned}
$$

Thus,

$$
D_{n}=a+\frac{1}{n+1}-a^{n},
$$

completing the proof.

\section{References}

[1] Bearden, J.N. (2006): A new secretary problem with rank-based selection and cardinal payoffs. Journal of Mathematical Psychology 50, 58-59.

[2] Bruss, F.T. (2005): What is known about Robbins' problem? Journal of Applied Probability 42, 108-120.

[3] Bruss, F.T. and T.S. Ferguson (1993), Minimizing the expected rank with full information. Journal of Applied Probability 30, 616-626. 
[4] de Carvalho, M., Chaves, L.M. and R.M. de Abreu Silva (2008): Variations of the secretary problem via game theory and linear programming. Journal of Computer Science 7, 78-82.

[5] Ferguson, T.S. (1989): Who solved the secretary problem? Statistical Science 4, 282-296.

[6] Gilbert, J. and F. Mosteller (1966): Recognizing the maximum of a sequence. Journal of the American Statistical Association 61, 35-73.

[7] Gnedin, A.V. and U. Krengel (1995): A stochastic game of optimal stopping and order selection. The Annals of Applied Probability 5, 310-321.

[8] Moser, L. (1956): On a problem of Cayley. Scripta Mathematica 22, 289-292. 